\title{
Digest the Lignocellulosic Degradation Mechanism in White-Rot Fungi to Guide the Design of Efficient Bioprocessing for Biomass Conversion
}

\author{
Su Sun, Jialong Zhang, Fei Li, Fuying Ma, Shangxian Xie, Xiaoyu Zhang* \\ Department of Biotechnology, College of Life Science and Technology, Huazhong University of Science and Technology, \\ 1037 Luoyu Road, Wuhan, 43004, China \\ sunsu@ hust.edu.cn; zhangjialong@hust.edu.cn; kasber-lee@163.com; mafuying@ hust.edu.cn; \\ shangxian_xie@hust.edu.cn; zhangxiaoyu@hust.edu.cn
}

\section{Extended Abstract}

White-rot fungi evolved with synergistic enzymes for efficient lignocellulosic biomass degradation, which offers an ideal model to reveal the mechanisms for lignocellulosic degradation. Understanding the white-rot fungal lignocellulosic deconstruction process is critical for designing efficient biomass conversion technologies. Multiple integrated "omics", comparative biomass structure analysis and enzymes characterizations were conducted to deeply digest the lignocellulosic degradation mechanisms in white-rot fungi Pleurotus ostreatus and Echinodontium taxodii. The multiple "omics" study in the white-rot fungi suggested that balanced and synergistic ligninase and polysaccharide hydrolysis enzymes complementing with efficient reactive radical generation were necessary for efficient lignocellulose degradation. The comprehensive surface property analysis, porosity measurements, and chemical structural characterization show that the lignocellulosic biomass after white-rot fungal decay achieved higher cellulose accessibility and lower biomass recalcitrance through reducing lignin and LCC content and modifying the chemical structures of lignin. The results suggested that selective removal of hydrophobic lignin coating cellulose increased substrate hydrophilicity and enlarged the volume of accessible pores of 5-10 $\mathrm{nm}$ diameter, which allowed more cellulase to infiltrate the lignocellulosic matrix to access the cellulose. The enzymes characterization further convinced the critical roles of manganese peroxidase, versatile peroxidase in lignin degradation and structural mediation, as well as their synergistic mechanism in lignocellulosic biomass degradation with other enzyme in the white-rot fungi. Typically, our result showed that $\mathrm{H} 2 \mathrm{O} 2$ generated by LPMOs was preferentially utilized for lignin-degrading peroxidase reaction, rather than cellulose oxidation indicating that white-rot fungi may have a strategy for preferential degradation of resistant lignin. Overall, our systemic study of the lignocellulosic biomass degradation process in white-rot fungi enabled us understand the mechanism of efficient biomass deconstruction to guide the design of efficient and costeffective bioprocessing for biomass conversion. 